\title{
NEUTRAL HYDROGEN IN COMPACT GALAXIES
}

\author{
J. HEIDMANN \\ Observatoire de Paris, Meudon, France
}

In collaboration with Messrs P. Chamaraux and R. Lauqué we have observed five objects of compact type or with compact parts at Nançay with the $21-\mathrm{cm}$ line of neutral hydrogen. Details will be found in Astron. Astrophys. 8, 424, 1970.

II $\mathrm{Zw} \mathbf{4 0}$ gives a well-defined line with nothing noticeable one beam East or West. I $\mathrm{Zw} 114$ and 17 give more poorly defined lines which may possibly be absent though the agreement of optical and $21-\mathrm{cm}$ radial velocities is in favour of their existences. Two are not detected.

All objects have low intrinsic luminosity. The three detected ones have sharp emission optical spectra and optical extensions.

Table I gives the main results:

TABLE I

\begin{tabular}{lccccc} 
Name & II ZW 40 & I Zw 114 & I Zw 17 & Arp gal. & Barbon gal. \\
\hline$M_{\mathrm{H}}\left(10^{8} \odot\right)$ & 2.3 & 1.0 & $<1.4$ & $<1.8$ & $<4.2$ \\
$M_{\mathrm{H} / L_{p}}$ & 0.3 & 1.0 & $<12$ & $<3.4$ & $<1.8$ \\
$\sigma_{\mathrm{H}}\left(\mathrm{km} \mathrm{s}^{-1}\right)$ & 55 & 35 & 40 & - & - \\
\hline
\end{tabular}

The hydrogen mass $M_{\mathrm{H}}$ is of the order of $10^{8}$ solar masses as for low luminosity irregular galaxies.

The hydrogen mass to luminosity ratio $M_{\mathrm{H}} / L_{p}$ is about unity as for late type galaxies.

The rms radial velocity dispersion of neutral hydrogen $\sigma_{\mathrm{H}}$ is of the order of $50 \mathrm{~km} \mathrm{~s}^{-1}$.

From the negative results obtained East and West of II $\mathrm{Zw} 40$, its $\mathrm{HI}$ E-W width at half intensity is smaller than $3^{\prime} .8$.

Using this upper limit, $\sigma_{\mathrm{H}}$ and the virial theorem, an upper limit of the total mass of II $\mathrm{Zw} 40$ is $10^{10}$ solar masses. Hence its mass to luminosity ratio is smaller than 15 and its hydrogen mass to total mass ratio is larger than 0.02 .

It is interesting to compare the $\mathrm{HI}$ velocity dispersions $\sigma_{\mathrm{H}}$ to the ones for stars $\sigma_{*}$. From its spectral resolution, Sargent gives $450 \mathrm{~km} \mathrm{~s}^{-1}$ as the upper limit for the emission lines in II $\mathrm{Zw} 40$ and I Zw 114. Values of $\sigma_{*}$ have been published for three other compacts by Minkowski and by Zwicky: $950,>700$ and $370 \mathrm{~km} \mathrm{~s}^{-1}$. The last value refers to NGC $4486 \mathrm{~B}$ which has a size and luminosity comparable to the objects which we observed; however its spectrum is a late absorption one.

If $\sigma_{*}$ in II Zw 40 were as large as for NGC $4486 \mathrm{~B}$, Kepler's law would lead to an $\mathrm{HI}_{\mathrm{s}}$ spatial extent for II $\mathrm{ZW}_{\mathrm{W}} 40$ a few tens times larger than the compact part size. The situation would be comparable to the one for normal galaxies from the points 
of view of $\mathrm{HI}$ extent and $\mathrm{HI}$ projected density and, because the stars are so much more concentrated, this would require a recent origin for this $\mathrm{HI}$ distribution.

But at this meeting Dr Searle kindly informed me that he recently obtained higher dispersion spectra of II $\mathrm{Zw} 40$ and I $\mathrm{Zw} 17$ and that, though their reduction is not yet completed, he thinks that the $\sigma$ values of the emission lines are comparable to our $\sigma_{\mathrm{H}}$ values.

If the emission line widths reflect the star velocity dispersions, taking $\sigma_{*}=\sigma_{\mathrm{H}}$ and using the $4^{\prime \prime}$ core size given by Barbon for II $\mathrm{Zw} 40$, the total mass of this part would be $3 \times 10^{8}$ solar masses. This value is comparable to the mass of very low luminosity irregular galaxies. It is comparable to the HI mass we observed. A large part of the mass of II $\mathrm{Zw} 40$ would then be made up of neutral hydrogen and the mass to luminosity ratio would be quite small, namely, 0.4 . The life time of such an object could not be very large. This raises interesting problems.

The origin of the neutral hydrogen is not clear: whether it was there for $10^{10} \mathrm{yr}$ before flashing up in stars, or whether it came from another object is difficult to say. We shall measure the $21-\mathrm{cm}$ line of II $\mathrm{Zw}_{\mathrm{w}} 40$ with a higher velocity resolution in order to gain kinematical information. Direct measurements of the $\mathrm{HI}$ distribution with a high resolution and high sensitivity radiotelescope, such as the Westerbork one, should bring precious data.

Evidently, compact objects with other characteristics should be observed with the $21-\mathrm{cm}$ line, with special emphasis on common optical and $21-\mathrm{cm}$ studies.

NGC $4486 \mathrm{~B}$ and the compact part of II $\mathrm{Zw} 40$ provide two nice illustrative examples of the ways in which an extragalactic stellar system can be compact, if by being compact we mean having a high surface brightness, as originally introduced by Zwicky. NGC $4486 \mathrm{~B}$ is compact through having much mass with a high $M / L$ ratio; the compact part of II $\mathrm{Zw} 40$ is compact through having little mass with a low $M / L$ ratio.

\section{Discussion of Papers Read by Dent and Heidmann}

Weliachew: I observed NGC 1068 about a year and a half ago with the transit telescope at Nançay, France. The limit I had for absorption was $1 \%$ instead of your $4.5 \%$. At any rate, the limits one then gets for the ratio of hydrogen mass to total mass cannot be said to be small for an Sb galaxy, owing to the very high dispersion of this ratio in a given morphological type.

Dent: I agree, there is only a suggestion that neutral hydrogen may be under-abundant in NGC 1068. In a paper yet to be given Dr Allen comes to a similar conclusion.

Weliachew: Are the absorption limits you quoted rms noise or rms noise multiplied by a certain factor?

Dent: They are peak-to-peak noise fluctuations.

Heidmann: Last year we observed the Seyfert NGC 4151 with the 21-cm line (Bottinelli et al.: 1970, Astron. Astrophys. 6, 453). This galaxy shows no peculiar behaviour. When plotted in the twodimensional diagram giving $\mathrm{HI}$ mass vs morphological type and intrinsic luminosity, the HI mass has a normal value and if one subtracts the light excess arising from the nucleus activity, this $\mathrm{HI}$ mass is even on the high side. Also, the mean mixed projected HI density is normal.

More particularly, the line width yields a maximum rotational velocity $V_{m}=115 \mathrm{~km} \mathrm{~s}^{-1}$, while its mean value for type 2 (Sab) galaxies is $200 \mathrm{~km} \mathrm{sec}^{-1}$. Then $V_{m}$ for NGC 4151 is rather on the low side. So:

(1) no HI deficiency, which might result for instance from ionization produced by activity in the nucleus, is observed in NGC 4151; 
(2) no high velocity expansion of large Hi masses, which might also result from such an activity, is observed.

Ekers: Observations of the QSO PKS0237-23 at the Owens Valley Radio Observatory showed no absorption due to $21-\mathrm{cm} \mathrm{HI}$ at a redshift of 1.36 , one of the optical absorption line redshift systems. The limit obtained was $<7 \%$ (2 standard deviations) in any of eleven $100 \mathrm{kHz}$ channels centered on $z=1.3642$ (frequency $600.8 \mathrm{MHz}$ ).

Searle: II Zw 40 and I Zw 17 have been observed optically by Sargent and myself. Absolute energy distributions and line intensities have been obtained by photo-electric scanning of the kind discussed by Oke earlier in this meeting.

The spectra, energy distributions, fluxes, absolute dimensions and surface brightnesses of these two 'compact galaxies' are very similar to those of the largest HII regions in external galaxies. Their spectra contain no indication of the presence of red stars and in this respect they differ from normal irregular galaxies. Our tentative conclusion is that these objects are intergalactic Hil regions or, equivalently, young dwarf galaxies. 